See discussions, stats, and author profiles for this publication at: https://www.researchgate.net/publication/268527647

\title{
Modeling leaf growth of rosette plants using infrared stereo image sequences
}

Article in Computers and Electronics in Agriculture · January 2015

DOI: $10.1016 /$ j.compag.2014.10.020

CITATIONS

26

6 authors, including:

$$
\text { Eren Erdal Aksoy }
$$

Halmstad University

48 PUBLICATIONS 629 CITATIONS

$$
\text { SEE PROFILE }
$$

Hanno Scharr

Forschungszentrum Jülich

116 PUBLICATIONS 2,562 CITATIONS

SEE PROFILE

Some of the authors of this publication are also working on these related projects:

Project Xperience View project

Project IntellAct View project
READS

209

Florentin Wörgötter

Georg-August-Universität Göttingen

461 PUBLICATIONS 6,433 CITATIONS

SEE PROFILE

Andreas Fischbach

Forschungszentrum Jülich

17 PUBliCATIONS 523 CITATIONS

SEE PROFILE 
In this paper, we present a novel multi-level procedure for finding and tracking leaves of a rosette plant, in our case up to 3 weeks old tobacco plants, during early growth from infrared-image sequences. This allows measuring important plant parameters, e.g. leaf growth rates, in an automatic and non-invasive manner. The procedure consists of three main stages: preprocessing, leaf segmentation, and leaf tracking. Leaf-shape models are applied to improve leaf segmentation, and further used for measuring leaf sizes and handling occlusions. Leaves typically grow radially away from the stem, a property that is exploited in our method, reducing the dimensionality of the tracking task. We successfully tested the method on infrared image sequences showing the growth of tobacco-plant seedlings up to an age of about 30 days, which allows measuring relevant plant growth parameters such as leaf growth rate. By robustly fitting a suitably modified autocatalytic growth model to all growth curves from plants under the same treatment, average plant 
growth models could be derived. Future applications of the method include plant-growth monitoring for optimizing plant production in green houses or plant phenotyping for plant research.

Keywords: leaf segmentation, leaf tracking, leaf modeling, plant growth, phenotyping

\section{Introduction}

With increasing requirements for food due to a growing world population, optimizing plant production is becoming an important factor for the agricultural industry. Plant performance and productivity results from a complex interaction between its genotype and environment, resulting in its expressed properties, i.e. its phenotype. Thus, if one seeks to understand these interdependencies, e.g. to achieve larger yields, plant phenotypes in terms of expressed plant structure and function need to be analyzed quantitatively. For this task automatic, non-invasive methods are highly desirable, but problems arise from the complex and varying appearance of plants, making it difficult to detect and recognize relevant plant organs and growth patterns.

Previously both color and stereo vision have been used to obtain some relevant plant features, mainly for recognition and classification purposes (Loch et al., 2005; Moeslund et al., 2005; Quan et al., 2006; Biskup et al., 2007; Song et al., 2007; Jin and Tang, 2009; Alenyà et al., 2011a; Teng et al., 2011; Silva et al., 2013; Wang et al., 2013), but those procedures are error prone, or require the concourse of a user to correctly segment and characterize individual leaves. For instance, Quan et al. (2006) modeled plants directly from a set of images for a better supervised leaf segmentation. Jin and Tang (2009) detected corn plants by only using depth images without dealing with the tracking issue. Leaf tracking has, to our knowledge, so far only been performed with unambiguously identified 
leaves. For example, Biskup et al. (2007) tracked the leaf orientation angles, and Polder et al.(2007) used penalized likelihood warping and robust point matching of leaf contours in order to detect emerging damages caused by disease. Alenyà et al. (2011b) showed how a robot arm can track a manually selected single leaf using some geometrical characteristics and color information. The problem of tracking multiple leaves was not addressed by these works. The work in (De Vylder et al., 2013) uses active contours to track multiple leaves, but they process time lapse plant images in batch once the complete sequence is acquired. Their proposed segmentation approach is triggered with the last frame of the sequence in a semi-supervised manner and the detection phase can omit new leaves since it goes to the first frame starting from the last one. De Vylder et al. (2011) combined active contours with a Bayesian framework to eliminate parameter tuning steps in the segmentation and tracking phases. However, they need manually segmented images to have a good estimate of the probability distribution functions for the calculation of internal and external probabilities. Both approaches (De Vylder et al. 2013,2011$)$ have also not been tested on plant sequences that last longer than 3 days.

Along this line, the European project GARNICS (ardening with a Cognitive System) $)^{1}$ aimed at 3D sensing of plant growth and building perceptual representations for learning the links to actions of a robot gardener (see Figure 11). The project encompassed both the long-term learning of treatments to achieve specific goals (maximum leaf growth, homogeneous plant growth) as well as the short-term robot interaction with plants (for leaf surface measurement, disocclusion, probing), and this study has been conducted in this context.

\footnotetext{
1http://www.garnics.eu
} 


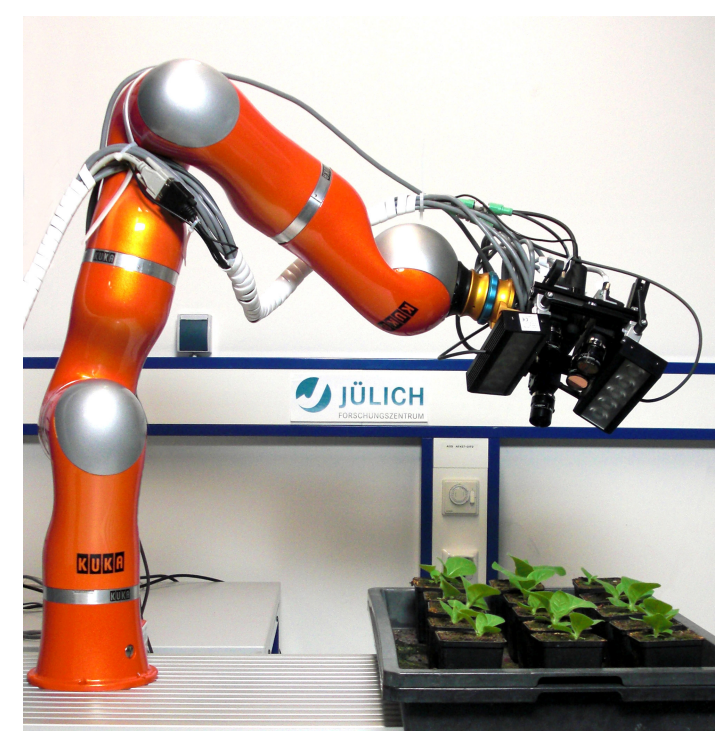

Figure 1: Robot gardener used in the European project GARNICS. A black-and-white 5 MP camera with infrared filter and required illumination devices were mounted on a lightweight KUKA LBR4 robot arm. For each tobacco plant the robot arm captured a stereo image pair from a top view at every hour.

More precisely, we address the problem of sensing and controlling plant growth parameters by ways of leaf tracking and model fitting, using a stereo infrared camera set-up, monitoring tobacco seedlings during their first three weeks of growth. A major difficulty hereby arises from the complex appearance of plants in the image. Leaves are weakly textured, often overlapping, thus occluding each other, and their form may be distorted in the $2 \mathrm{D}$ projection due to steep leaf angles with respect to the camera view. Under these conditions, the automated image segmentation of individual leaves is highly challenging, and cannot be guaranteed. In this work, we first over-segment the infrared images and then employ a merging procedure using a 2D leaf-shape model, but also incorporating $3 \mathrm{D}$ information from stereo matching. The main growth curves of the plant leaves are extracted and used to analyze plant development over time. Segmentation failures appear as noise in the system, and can be handled at least to some degree. Once the main growth curves 
corresponding to the individual leaves of the plant are found, erroneous segments can be removed, and by using a leaf-shape model, the growth rates for each identified leaf can be computed.

Rosette plants are commonly used in plant research facilities, and the automatic growth analysis of seedlings would come in handy for many laboratories. Furthermore, growth monitoring of seedlings can be used in plant production to optimize plant treatments, e.g. with respect to the provision of water and nutrients or light requirements. Size and color distribution of plant leaves over time are important cues to monitor the lack of such requirements, avoiding plant stress situations.

Note that this study has also been described as a part of a patent (Wörgötter et al. 2013).

\section{Plant Material}

Six tobacco plants (Nicotiana tobacum cv. Samsun) were grown under constant light conditions ( $500 \mu \mathrm{E} \mathrm{m}^{-2} \mathrm{~s}^{-1}$ ) with a $16 \mathrm{~h} / 8 \mathrm{~h}$ day/night rhythm. Three of them (Plant IDs 79329, 79335, and 79338) received $1.8 \mathrm{ml}$ of water every other hour (“Treatment 1"), the others (Plant IDs 79330, 79336, and 79339) received $0.9 \mathrm{ml}$ of water and $0.5 \mathrm{ml}$ of nutrient solution with $1 \%$ Hakaphos green every other hour ("Treatment 2"). Water and nutrient solution were applied by the GARNICS robot system, positioning small tubes, one for water and one for nutrient solution, at predefined locations and pumping using an automated flexible-tube pump.

In the GARNICS project, treatments were selected to produce training data for a cognitive system. The actual amounts of water and nutrient solution are therefore well adapted 
to the soil substrate such that the sets of plants show distinguishable performance of generally well growing plants. Finding an optimal treatment was left for the system. The soil used for the experiment ("Kakteenerde") has low nutrient content and dries relatively fast with an approximately exponential behavior $A=A_{0} \exp (-t / \tau)$, where $\tau \approx$.7days.

We applied the proposed leaf tracking and modeling algorithm to tobacco-plant sequences showing the growth from germination well into the leaf development stage, i.e. we started our observations at growth stage 09 and typically stopped at stage 1006 (according to the extended BBCH-scale presented in CORESTA (2009)), due to size restrictions.

\section{Method}

\subsection{Overview}

Our framework for continuous measurement of plant growth parameters consists of three main parts: data acquisition and preprocessing, segmentation of all frames from a plant video sequence, and consistent leaf tracking and modeling of the segmented leaves. A schematic showing all steps of the procedure and labeled by numbers is presented in Figure 2 .

As input data we use gray-scale stereo images acquired with an infrared camera attached on a robot arm. We compared different illumination options and found that plant structures and boundaries between tobacco leaves could be detected more easily for infrared light than for visible light. In addition, plants do not react to the applied 880nm IR light, e.g. by photosynthetic activity. Consequently, illumination and acquiring images at night is possible without influencing plant growth, in contrast to visible light. A pair of images (left and right) is captured at each time step by moving the robot head with the 


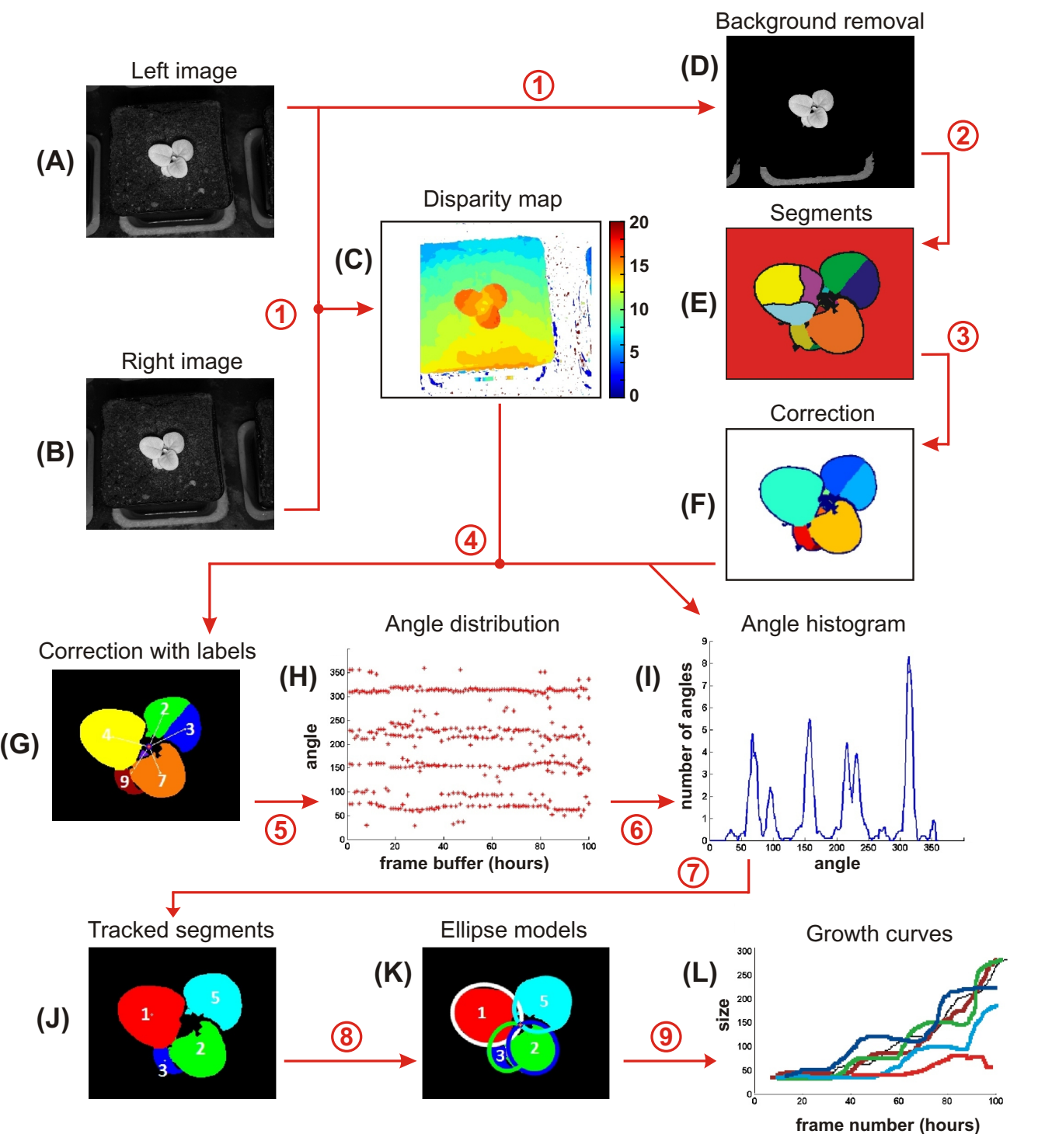

Figure 2: Schematic of the multi-level procedure for segmenting and tracking leaves. Numbers mark the different computational steps of the procedure. (A) Acquired left frame. (B) Acquired right frame. (C) Disparity map estimated using block matching. (D) Left frame after background removal. (E) Image segmentation results using the method of superparamagnetic clustering of data (here and further scaled up for a better visibility). (F) Segments after segment merging, and (G) after relabeling. (H) Angle distribution of corrected segments for 100 frames. (I) Angle histogram derived from the angular distribution. (J) Tracked segments with reassigned unique labels. (K) Ellipse models fitted to the tracked segments. (L) Plant growth curves estimated from ellipse models. 
infrared camera and light source, providing a stereo baseline (see Figure 2(A) and (B)). In step 1 of the procedure, we compute a depth (disparity) map from the stereo pair using a block-matching algorithm from the OpenCV library Bradski (2000) (see Figure 2(C)). This method gave preferable results compared to other methods. We further removed the background from the scene to simplify the following computations (see Figure 2(D)).

Next, in step 2, each preprocessed infrared image of the sequence is segmented independently. Afterwards, each leaf is represented by one or more segments as shown in Figure 2(E). In step 3, we employ a merging procedure to group the segments into leaf shapes (Figure $2(\mathrm{~F})$ ) by finding the partition that minimizes the overlap between the convex hulls of the segments. This is a good enough working assumption as long as the leaves have convex shapes. This merging stage is a necessary improvement, but it still does not guarantee success. Sometimes there are over-segmentations which remain unresolved, as shown in Figure $2(\mathrm{~F})$. Note that after merging, the segments are relabeled (see Figure $2(G))$.

In step 5 of the procedure, the position of the centroid of each segment is computed with respect to the plant stem position in polar coordinates. The plant stem can be found with sufficient accuracy by computing the centroid of the foreground (containing only the plant) at an early growth stage. By presenting each segment as a data point in an angletime plot, growth tracks can be made visible because the tobacco-plant leaves do hardly change their azimuthal angle (Figure $2(\mathrm{H})$ ). Leaves that are growing in the same direction can be distinguished based on their depth values. Hence, when computing the angular histogram of the centroids over a larger time interval (step 6 of the procedure), the data points of the growth tracks accumulate at the angular positions of the corresponding leaves 
(see Figure 2(I)). By first detecting the peaks in the histogram using a threshold, we can cluster the segments belonging to the different tracks and assign them unique, temporally consistent labels in step 4 (see Figure 2(J)). In the final step (9), tracked segments, corresponding to leaves, are used for fitting appropriate ellipse models (see Figure $2(\mathrm{~K})$ ) and estimating growth curves for individual leaves as shown in Figure 2(L).

\subsection{Image Acquisition}

For image acquisition a black-and-white $5 \mathrm{MP}$ camera with infrared pass filter has been used. Images have been taken at regular, hourly time intervals for each plant over a time period of 30 days. The camera was mounted on a lightweight KUKA LBR4 robot arm (see Figure 1). For each plant the robot arm captured a stereo image pair from a top view every hour by moving a certain distance (app. $5 \mathrm{~mm}$ ) along the baseline.

\subsection{Preprocessing}

Before segmenting the images, we remove the background as shown in the second row of Figure 3. The table, the plant pot, and the soil visible in the near infrared (NIR) images can be easily eliminated by applying a threshold. Furthermore, a disparity map is computed with a standard block-matching technique from the stereo infrared images.

\subsection{Leaf Segmentation}

For segmenting the images, we use the method of superparamagnetic clustering of data which runs in real-time on a Graphics Processing Unit (GPU). The method of superparamagnetic clustering is inspired by systems of interacting ferromagnets or spins. These systems are characterized by three phases. At low temperatures, spins are fully aligned with one another, while at intermediate temperatures, groups of aligned spins coexists. At 
higher temperatures, the order breaks down into a disordered state. When representing pixels by spins and defining spin-spin interactions dependent on the similarity of adjacent pixels, a natural partition of the image can be found in the superparamagnetic regime simulating the stochastic dynamics of the system with a Metropolis algorithm.

The method of superparamagnetic clustering has been described in detail elsewhere Abramov et al. (2012). Superparmagnetic clustering has been used previously to segment leaves based on color and depth acquired with a Kinect camera (Wallenberg et al. (2011)). However, in this case, plants were fully grown and leaves considerably larger. In our experimental set-up, leaves are smaller, and the task of obtaining sufficiently accurate depth information for depth-based segmentation would be far more challenging. Typical segmentation results obtained by this technique are shown in the last row of Figure 3 .

Due to varying light conditions and very low intensity differences at the leaf borders,
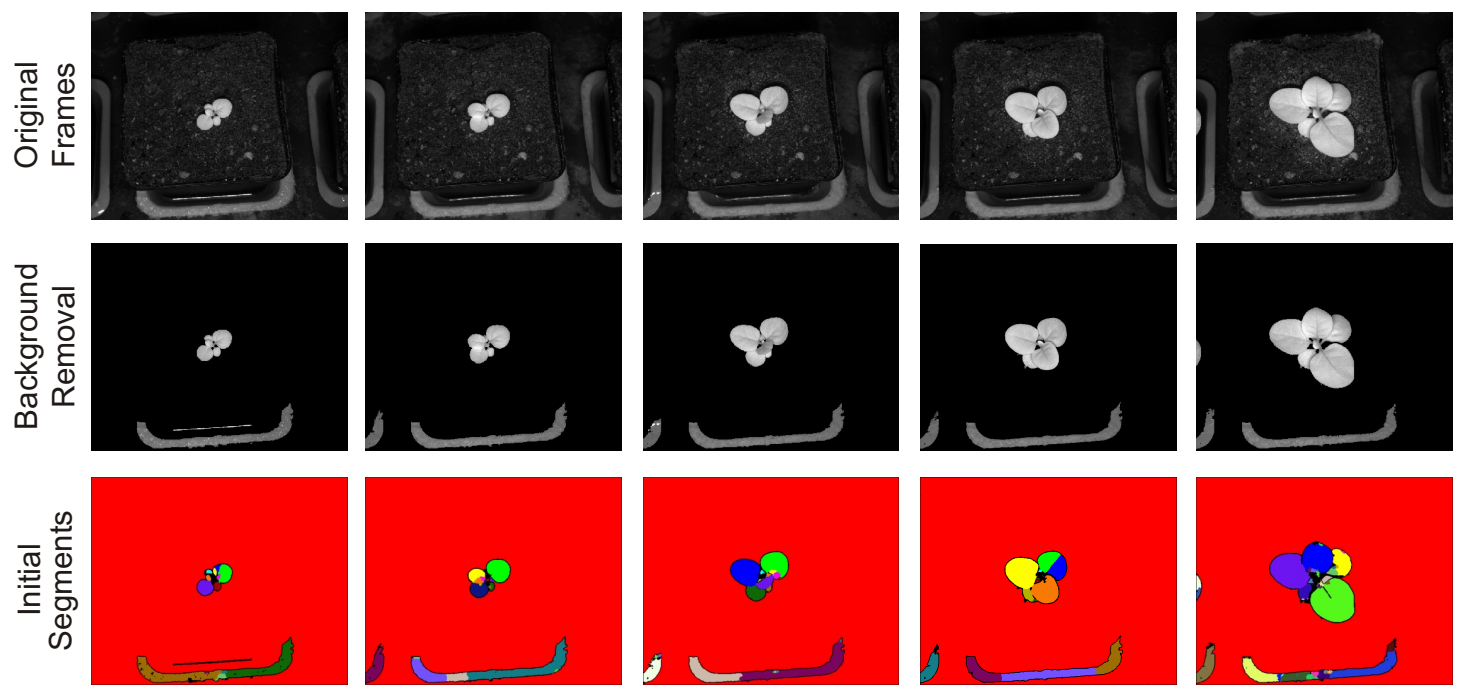

Figure 3: Segmentation of near infrared (NIR) images using the method of superparamagnetic clustering. First row shows left input frames captured with an infrared camera, middle row indicates frames after the background removal, and the last row shows initial segmentation results (after step 2 of the procedure). 
leaves may be wrongly merged by the method. To avoid this, the segmentation runs in the over-segmentation mode (see Figure 3). This strategy ensures that segments adhere better to leaf borders. Leaves represented by more than one segment can be recovered later on (see Section 3.5), while recovery of two (or more) leaves from one big wrongly merged segment (under-segmentation) is more difficult.

\subsection{Segment merging}

The output given by the image segmentation module many times splits one leaf into more than one segment and may contain noisy regions, such as a part of the pot or some areas of high intensity compared to the background. Therefore, additional procedures are required in order to obtain a better segmentation. The first major improvement is achieved by correcting the initial segments with a leaf-shape descriptor. For this purpose tobacco plant leaves can be described by their convex hulls with sufficient accuracy.

The segment-merging procedure works as follows. First of all, segments with centroids located far from the plant stem are eliminated (see the first row in Figure 4). Noisy speckles are removed as well (see the second row in Figure 4). Then a graph is built where the centroids of the segments represent the graph nodes. Edges are drawn between two nodes if the segments are smaller than a threshold apart both in $(x, y)$ distance and depth. Each edge represents a possible merge. Hence, for a total number of $s$ edges, there are $2^{s}$ possible merging configurations $M_{i}$. Neglecting occlusions, the desired segmentation should more or less preserve the shape of the leaves, i.e., using the segment's convex hull as leaf-shape model, the total overlap of the convex hulls of all segments should be smallest for this configuration. Let now be $C_{j}$ the convex hull of segment $j$, then we compute the overlap of a particular merging configuration $M_{i}$ as $O_{i}=\sum_{e_{l m} \in M_{i}} C_{l} \cap C_{m}+\sum_{k} C_{k} \cap B$, 

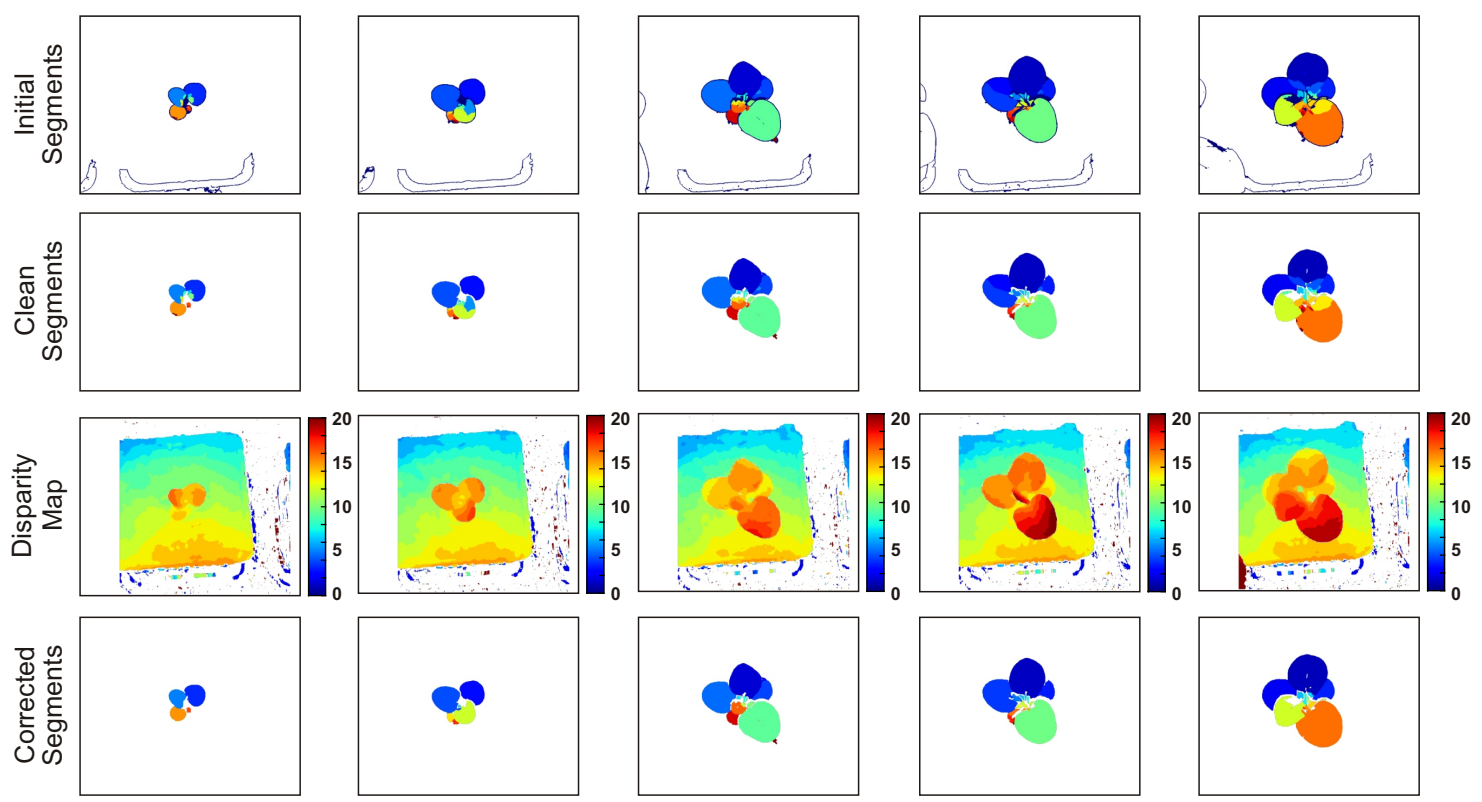

Figure 4: Segment correction performed by the convex hull approximation with depth information. Top row shows initial segments. Second row indicates clean input segments without noise and borders. Third row represents disparity maps estimated by the block matching technique for a pair of NIR images. Final segments after segment merging are shown in the last row (after step 3 of the procedure).

where $B$ is the background region. We select the merging configuration with the smallest overlap. For a small number of edges, we can simply evaluate all possible configurations. This is the case in our scenario. For large number of edges, approximate methods would have to be employed to find the minimum.

The depth data (see the third row in Figure 4) is used to remove edges between neighboring segments that have a large difference in disparity. This also helps to keep $s$ reasonably small. Typical results of the segment-merging procedure segmentation are shown in the last row of Figure 4.

Merging segments that represent a leaf based on shape features is a difficult problem for the following reasons: Only a small part of the boundary of a leaf segment corresponds 
to the actual leaf boundary (the other ones are inner boundaries, i.e, non-leaf boundaries). Pairwise merging, as employed in standard split-and-merge approaches, will thus only be successful for simple cases because the shape of the whole leaf will only become apparent when all the segments have been merged correctly and all inner boundaries have been removed through the merging process. This is a typical chicken-egg problem. Occlusion adds further difficulties by altering the visible shape of the leaves. For this reason, given the small number of segments, we opted for the described merging technique which avoids having to apply a standard pairwise merging procedure (Horowitz and Pavlidis (1974); Aleny $\tilde{A}$ et al. (2013)) and instead tests for all possible combinatorial solutions.

\subsection{Tracking}

Usually leaves grow at an almost constant azimuth angle with respect to the plant stem during their development, and even if two leaves have the same angle, their depth values typically are different. Therefore, we can make use of the natural growth pattern of plant leaves for solving the tracking issue.

For each frame, we first calculate coordinates of the plant stem $p=\left\{p_{x}, p_{y}\right\}$ as

$$
p_{x}=\frac{1}{N} \sum_{i=1}^{N} s_{x_{i}} \quad, \quad p_{y}=\frac{1}{N} \sum_{i=1}^{N} s_{y_{i}}
$$

where $N$ is the total number of existing segments, whose centers are given by $\left\{s_{x}, s_{y}\right\}$.

Each segment center is then represented by $r$ and $\theta$ defining the radius and angle in polar coordinates as

$$
r=\sqrt{\left(s_{x}-p_{x}\right)^{2}+\left(s_{y}-p_{y}\right)^{2}} \quad, \quad \theta=\arctan 2\left(\frac{s_{y}-p_{y}}{s_{x}-p_{x}}\right)
$$


At each acquired frame, all extracted $N$ segment angles are combined into a histogram $H$ representing the distribution of angles over previous $T$ frames as

$$
\begin{gathered}
H=\left\{h_{i}: i \in\left[1,2, \cdots, \frac{360}{k}\right]\right\} \\
h_{i}=\sum_{n=1}^{N} \sum_{t=1}^{T} \delta_{n, t}, \\
\delta_{n, t}= \begin{cases}1 & \text { if } i-1<\frac{\theta_{n, t}}{k}<i \\
0 & \text { else }\end{cases}
\end{gathered}
$$

where $k$ is the bin size. In our experiments $k$ and $T$ values are chosen as 10 and 100 unless otherwise stated. Fig. 5 (top row) shows four plant images. The corresponding segments from the merging procedure are shown in the second row. The respective angular distributions of their centroid positions over 100 frames are plotted in the third row of Fig. 5. The resulting histogram representation for each plant image is depicted in the fourth row in Fig. 5 .

We further continue with calculating local maxima (i.e. peaks) in each histogram and use them to cluster the data. Let $m_{i}$ and $m_{j}$ be the angle positions of two local maxima derived from a given angle distribution. The maximum at $m_{j}$ is basically ignored if $m_{i}-$ $m_{j}<\tau_{d}$, where $\tau_{d}$ is a threshold. In our experiments, we use $\tau_{d}=40^{\circ}$. The extracted local maxima (i.e. all $m_{i}$ ) are shown as red circles in Fig. 5. All other local maxima (i.e. all $m_{j}$ ) 

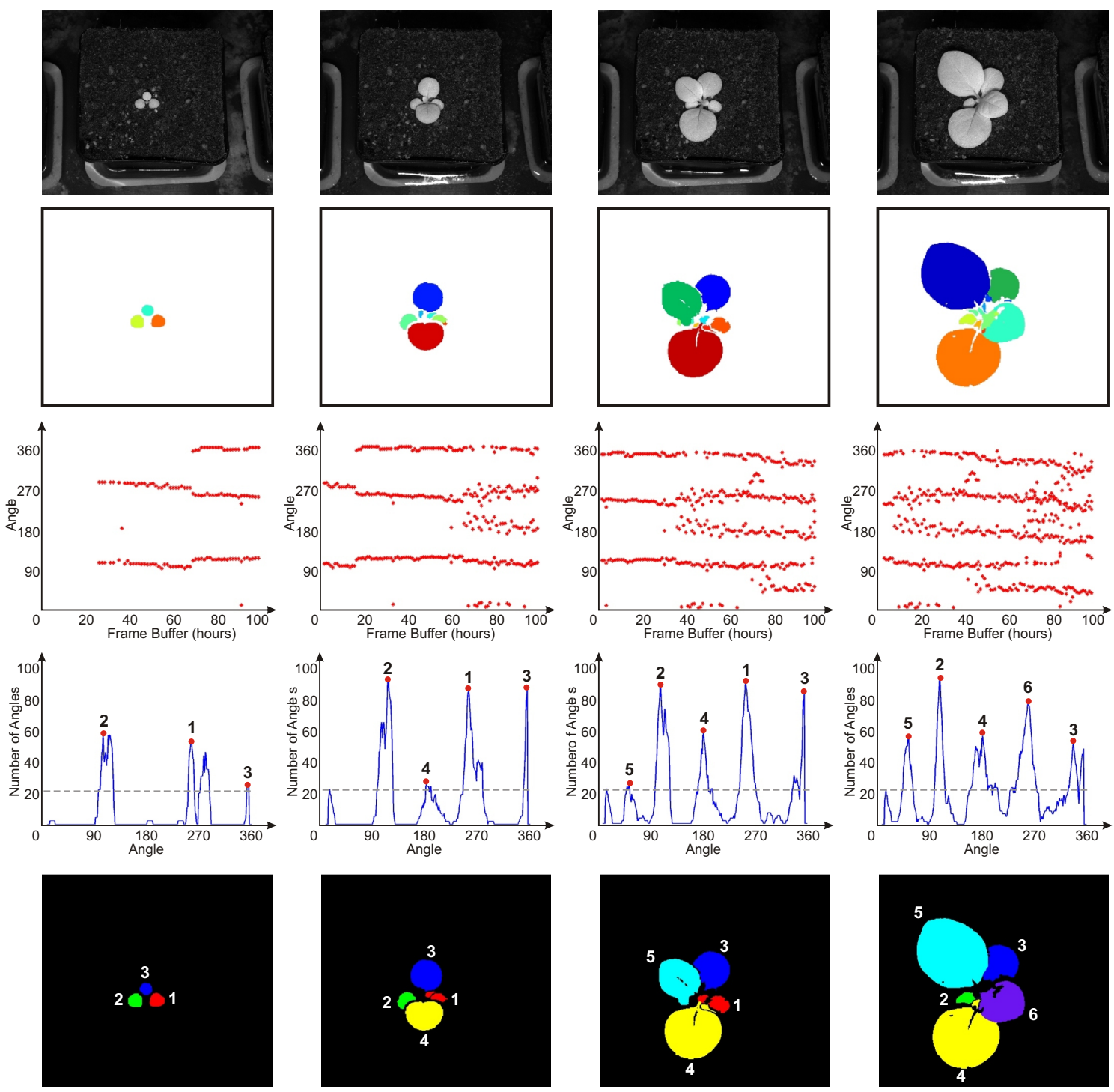

Figure 5: Tracking plant leaves with segment angles. Top row shows sample original plant images with corresponding corrected segments depicted in the second row. Segments are here scaled up for a better visibility. Respective angular distribution of segments over 100 previous frames are illustrated in the third row. Histogram representation of each distribution is depicted in the fourth row. Circles in red indicate calculated final local maxima with assigned unique labels. Dashed lines show the threshold values for local maxima. Last row indicates the final tracked leaf segment labels with their unique labels. 
are ignored since their distances to their nearest neighbors are below threshold. We also ignore those maxima which are smaller than the average histogram value

$$
\tau_{m}=\frac{k}{360} \sum_{i=1}^{\frac{360}{k}} h_{i}
$$

The threshold value $\tau_{m}$ for each histogram is shown as dashed lines in the fourth row of Fig. 5 .

The tracking phase is concluded by reassigning a new unique label $l_{i}$ for each maxima $m_{i}^{f}$ at frame number $f$. The label $l_{i}$ is transfered to another local maximum $m_{j}^{f+1}$ in the next frame $f+1$, if those maxima are neighbors within a certain threshold $\tau_{d}$ such that $\left|m_{i}^{f}-m_{j}^{f+1}\right|<\tau_{d}$. In this way, the final label-maxima correspondence map is updated at each frame to track segments continuously. In Fig. 5 (fourth row) the assigned labels corresponding to the extracted local maxima (indicated by red circles) are displayed. The first image shows the plant with three leaves, i.e. the cotyledons and first true leaf, then three more leaves appear one after the other.

During the tracking phase, the disparity values of corrected segments are used to distinguish leaves overlapping one another as shown in the last column of Fig. 5. Here, a new leaf, assigned with label 6 , is appearing and occluding the leaf with number 1 . In this case, these two leaves have almost the same angle, however, due to the differences in their disparity values, a new label can be assigned to the leaf. The final segmentation result is shown in the last row of Fig. 5 . 


\subsection{Leaf modeling and extracting leaf-growth curves}

Since leaves can occlude each other, the size of the tracked segments extracted using the methods described in the previous section cannot be used directly to estimate plant growth parameters. To address weak to medium occlusions we fit an ellipse model defined as $\xi=\{\mathcal{C}, \Theta, \mathcal{H}, \mathcal{W}\}$, where $\mathcal{C}, \Theta, \mathcal{H}$, and $\mathcal{W}$ represent ellipse center position, tilt angle, and the lengths of the major and minor semiaxes (height and width), respectively, to each tracked segment.

In order to calculate these ellipse parameters, we first determine each leaf tip position $\mathcal{T}$, i.e., a segment point with the maximum distance to the plant stem, from $N$ segment edge points $\left(e_{x}, e_{y}\right)$ as

$$
\begin{gathered}
\mathcal{T}=\left\{\mathcal{T}_{x}, \mathcal{T}_{y}\right\}=\underset{i}{\arg \max _{i}\left(d_{i}\right),} \\
d_{i}=\sqrt{\left(e_{x_{i}}-p_{x}\right)^{2}+\left(e_{y_{i}}-p_{y}\right)^{2}}, \quad i \in[1, \cdots, N],
\end{gathered}
$$

where $p_{x}$ and $p_{y}$ are the plant stem coordinates given in Eq. (11). We can now calculate the ellipse centroid coordinates $\mathcal{C}=\left\{\mathcal{C}_{x}, \mathcal{C}_{y}\right\}$ as,

$$
\mathcal{C}_{x}=\frac{\mathcal{T}_{x}+p_{x}}{2} \quad, \quad \mathcal{C}_{y}=\frac{\mathcal{T}_{y}+p_{y}}{2}
$$




$$
\Theta=\arctan 2\left(\frac{\mathcal{T}_{y}-p_{y}}{\mathcal{T}_{x}-p_{x}}\right) \quad, \mathcal{H}=\frac{\sqrt{\left(\mathcal{T}_{x}-p_{x}\right)^{2}+\left(\mathcal{T}_{y}-p_{y}\right)^{2}}}{2} \quad, \mathcal{W}=\frac{1}{N} \sum_{i=1}^{N} d_{i}
$$

where $d$ is the distance of $N$ segment edge points to the plant stem and is given in Eq. (6). Leaf area is then computed from the respective ellipse size depending on $\mathcal{H}$ and $\mathcal{W}$ values. Fig. 6 shows an example how segments are corrected, labels tracked, and ellipses fitted. In the top row, individual segmentations after segment merging (step 3) of the method are presented. The second row shows segments with reassigned labels after the tracking process has been completed (steps 5-7). The last row shows the ellipse models fitted to each segment. A movie with derived segments and ellipse models can be found at www.dpi.physik.uni-goettingen.de/ eaksoye/GARNICS. Fig-
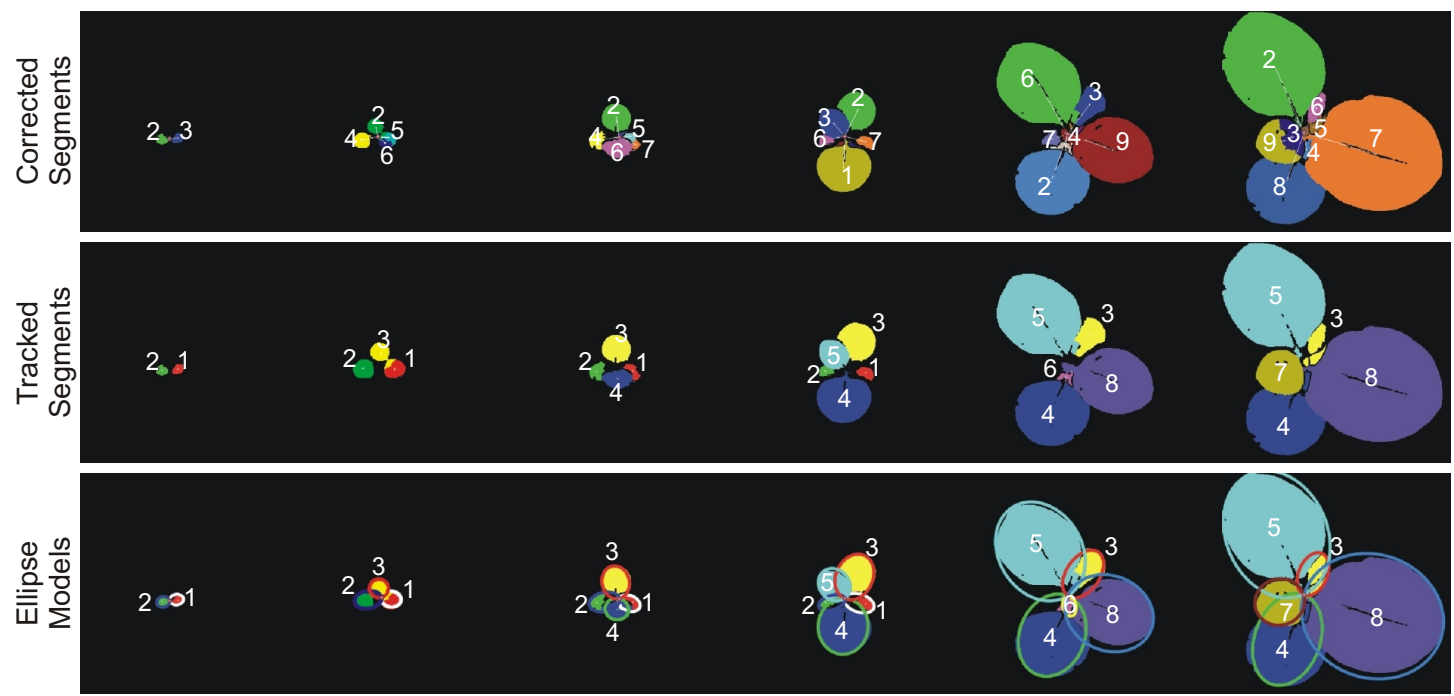

Figure 6: Leaf modeling with ellipses. Top row shows sample frames with corrected segments. Second row depicts corresponding tracked segments with reassigned unique labels. Here, each segment color represents one unique label. Last row is with final ellipse models estimated for each tracked leaf. 
ure 7 shows ellipse tracking results for all six plants.

Our leaf modeling approach is a searching-based method and there exist similar works in the literature (Song and Wang, 2007; Kaewapichai and Kaewtrakulpong, 2008). Chien et al. (2011) proposed an alternative ellipse detection framework which applies elliptical Hough transform to different levels in the image pyramid. Although this approach is robust
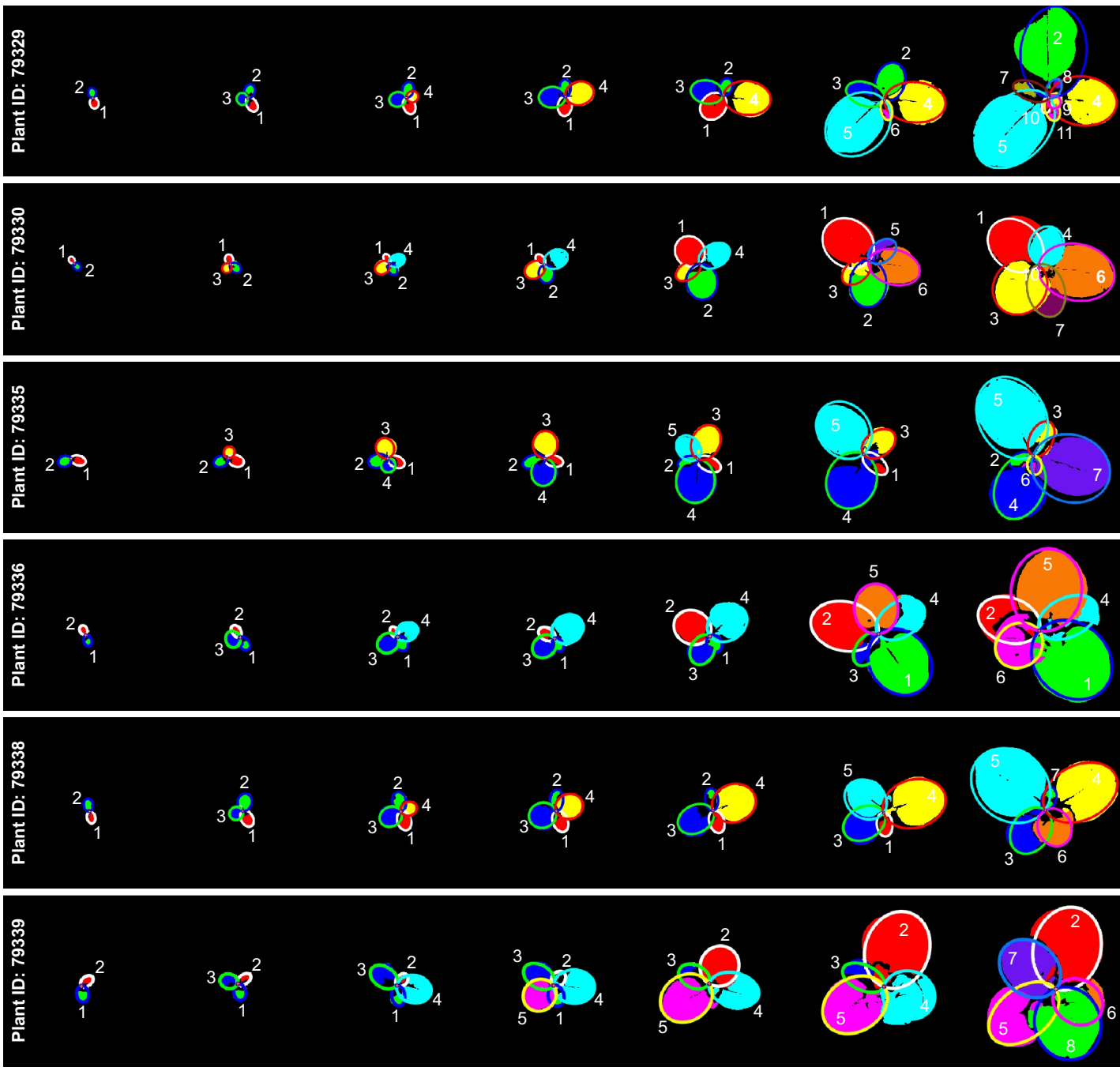

Figure 7: Tracking results and estimated ellipse models for six different tobacco plants. 
to noise during the extraction of multiple ellipses, it cannot be applied to our plant image sequences since small leaf segments, observed in the first weeks of the seedling, can vanish in the coarsest resolution in the image pyramid. Thus, detection of leaves can be delayed in the temporal scale.

\subsection{Resolving total occlusion}

In some cases, we observed that disparity and angle cues from Section 3.6 are not enough to distinguish between leaves. When a leaf is completely occluded by a subsequently appearing leaf, the first leaf's growth curve is sometimes continued by the second leaf. See e.g. Figure 7)Plant ID: 79330: Cotyledons (segments 1 and 2 in red and green, respectively) grow to a small size as expected, but in the $5^{\text {th }}$ and following depicted time instances seem to grow strongly. Same is true e.g. for Plant ID 79336. Fortunately this can be easily detected and corrected when plotting growth curves in terms of ellipse sizes as a function of time (cmp. log-plots of the growth curves in Figure 8).

The raw data (Figure 8 top left) is median filtered with a filter length of $24 \mathrm{~h}$ in order to suppress diurnal variations. Subsequently it is smoothed and small gaps interpolated by normalized convolution Knutsson and Westin (1993) using a Gaussian kernel with standard deviation $9 \mathrm{~h}$, length $27 \mathrm{~h}$ (Figure 8 top right). The resulting smooth curves are filtered to be monotonically increasing by processing them in positive time direction, keeping a vale if its is the current maximum, else replacing the current value by the so far seen maximum (Figure 8 bottom left). This enforces the assumption that leaves are not shrinking. These smooth monotonic curves are then cut into separate curves at gaps (cmp. Figure 8 bottom left, black lines, with the corresponding lines in Figure 8 bottom right), or when an almost non-growing part is followed by a strongly growing one (cmp. Figure 8 bottom 

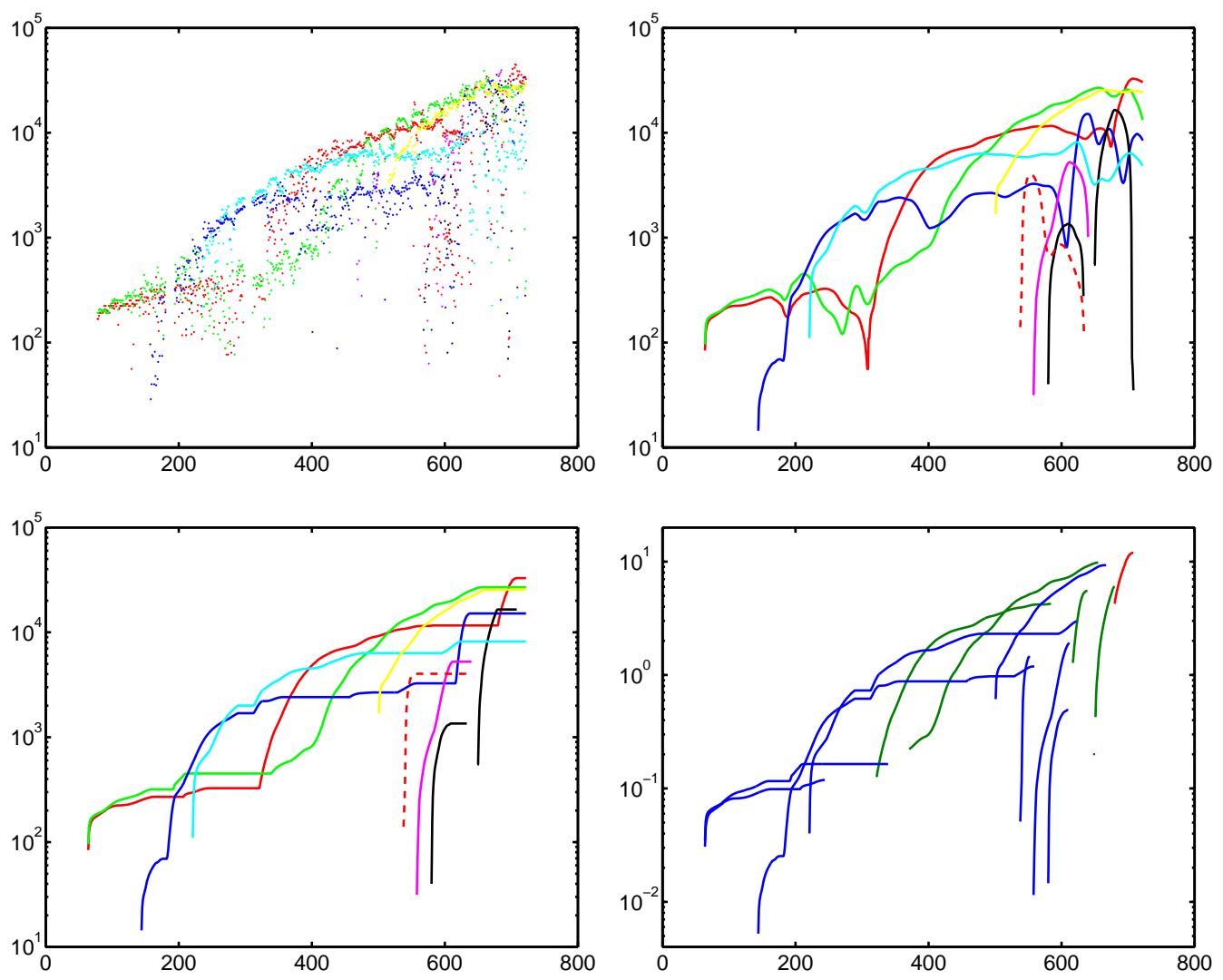

Figure 8: Measured leaf size versus time for Plant ID 79336. Top left: raw data. Different colors indicate different growth curves. Same is true for the next 2 plots. Top right: smoothed by median filter and gaps closed by normalized convolution. Bottom left: filtered to ensure monotonic increase. Bottom right: Growth curves split into curves belonging to a single leaf, horizontal beginnings and ends removed. Blue indicates the first section of a growth curve, green the second and red the third section stemming from one initial growth curve. The vertical and horizontal axes represent leaf size $\left(\mathrm{cm}^{2}\right)$ and time (hours).

left, e.g. red and green lines, with the corresponding lines in Figure 8 bottom right). At each curve, initial or trailing horizontal parts are removed, as they do not reliably reflect measurements, but extrapolations, only.

Due to the curve cutting process, the natural emergence order, i.e. that growth curve $n$ belongs to leaf $n$, is no longer given. Ideally curves should be sorted by the times when leaves have a certain, predefined size. This is not possible here, as some curves start at 

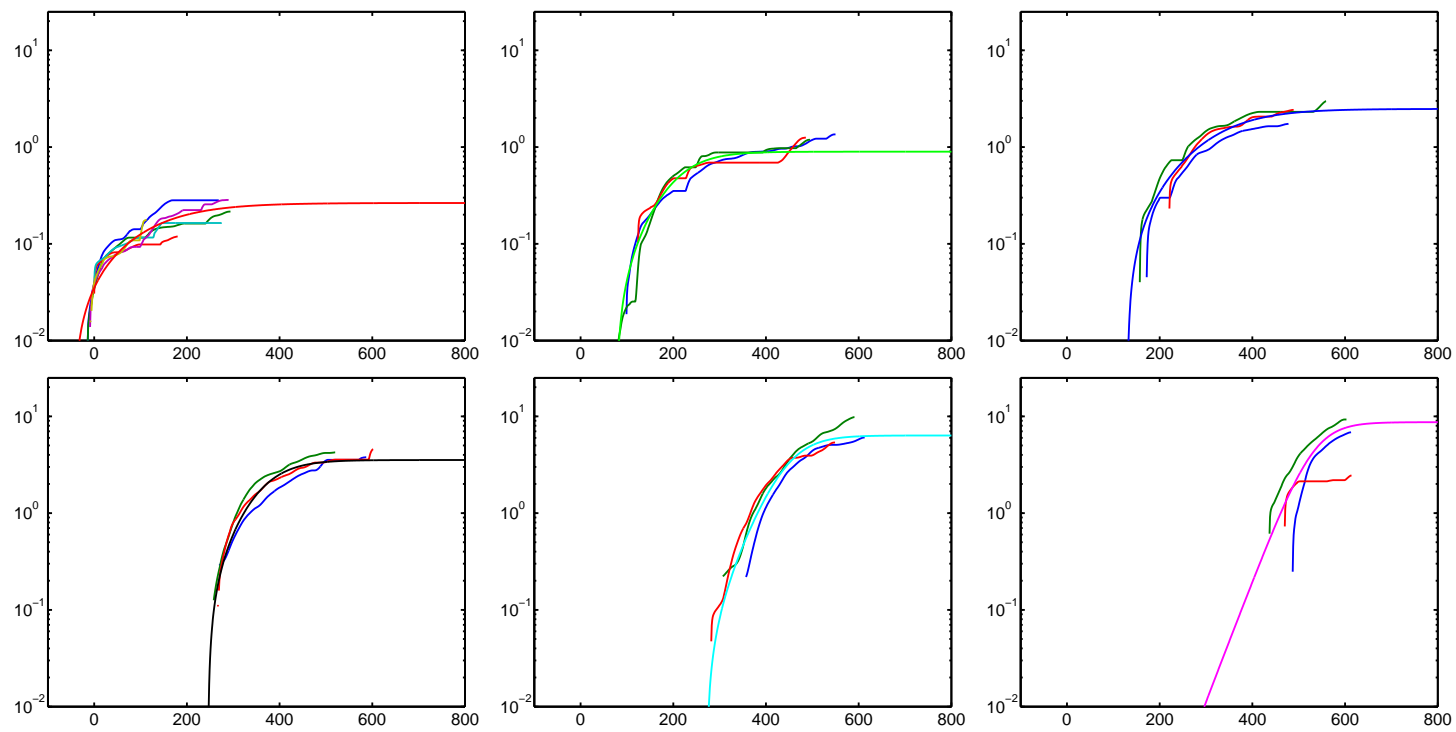

Figure 9: Mean growth curves for the treatment with more nutrients and less water (treatment 2): Single leaf growth curves of all 3 plants sorted by time of emergence, i.e. leaf number. Thick curves are the measured data, fine curve is the autocatalytic model with constant y-offset robustly fitted to all curves simultaneously. Left to right and top to bottom: cotyledons (i.e. leaf 1 and 2, being 6 curves for 3 plants), leaf 3 to 7 . Cotyledons are temporally aligned such that size $0.04 \mathrm{~cm}^{2}$ corresponds to time $0 \mathrm{~h}$. The vertical and horizontal axes represent leaf size $\left(\mathrm{cm}^{2}\right)$ and time (hours).

quite large leaf sizes. As sorting by emergence time of the curves would lead to wrong ordering, we compensate the later emergence of a growth curve by fitting a tangent in logscale (i.e. a purely exponential growth curve) to each curve and order by their time offsets. We use a high growth rate of $10 \% / \mathrm{h}$ for the tangent, being adequate due to a measurement offset (cmp. Section 3.9). The resulting growth curves sorted per leaf number of plants from the treatment with more nutrients and less water are depicted in Figure 9. There curves are temporally aligned such that the time point when the first Cotyledon reaches size $0.04 \mathrm{~cm}^{2}$ corresponds to time $0 \mathrm{~h}$. 


\subsection{Leaf growth modeling}

To each of the leaf-number-wise sorted growth curve groups (cmp. Figure 9) a growth model is fitted in a robust way (thin lines in the plots). We use the well known autocatalytic growth model (see e.g. Richards (1959)) with a slight modification

$$
A(t)=A_{\infty}(1+\exp (-g r(t-\tau)))^{-1}-A_{o f f s e t}
$$

where $A(t)$ is the leaf size at time $t, A_{\infty}$ is the final leaf size, $g r$ is the growth rate, $\tau$ is a time offset. $A_{o f f s e t}$ is an offset compensating an apparent slight underestimation of the true leaf size.

This model is fitted to the data using a robust error norm able to ignore outliers at a constant high cost. We use a variant of the truncated quadratic (Blake and Zisserman (1987)) where the constant cost after truncation is 10 times higher than the cost at the truncation limit. By this we ensure to have a maximum amount of inliers as e.g. required as optimality condition in random sample consensus (RANSAC, see Fischler and Bolles (1981)).

The time offset $\tau$ models the leveling off of the growth curve and is not suitable to estimate leaf emergence. Following Tsai et al.(1997) we use the time point $t_{c}$ when a leaf reaches a small given size $A\left(t_{c}\right)=c$. For our autocatalytic model we derive

$$
t_{c}=\tau-\frac{1}{g r} \log \left(\frac{A_{\infty}}{c+A_{o f f s e t}}-1\right)
$$




\section{Results}

\subsection{Fitted leaf growth models}

As we are here dealing with a system to measure early plant growth, we have investigated and modeled only the first few leaves (counting cotyledons as leaves 1 and 2). When plants are getting bigger, we observe large and rapid variations in the size estimates for some leaves. This is because wrong segment and depth estimations occur more often during this phase. Thus measurements become less reliable making leaf sorting ambiguous. In Figure 10 we therefore show results for the first 7 leaves, only. Looking at fitted final leaf size $A_{\infty}$ for the averaged plant models we observe, that plants under Treatment 1 (see Section 2) grow much larger leaves than under Treatment 2. However, not only growth rates $g r$ are higher, but also the time span $\tau-t_{c}$ between leaf "emergence" $t_{c}$ and leveling off time $\tau$. For Treatment 1 the average growth duration is $114 \mathrm{~h}$, for Treatment 2 it is $99 \mathrm{~h}$.

The estimated phyllochron, i.e. the time between leaf "emergence" time points $t_{c}$, varies also slightly, average $65 \mathrm{~h}$ for Treatment 1 and $61 \mathrm{~h}$ for Treatment 2 . Leaf 3 , the first leaf after the cotyledons, emerges after 2 to 3 days after these. Leaf 4 then emerges quicker (1.5 to 2 days) and leaf 5 then takes 5 to 6 more days to emerge. Leaves 6 and 7 then again emerge quicker after 2 to 3 days. Thus for our small dataset we observe that there is no constant time interval between emergence of leaves, but leaf 5 emerges with a considerable delay for both treatments.

\subsection{Benchmarking the method}

The functioning of the framework presented in this paper strongly depends on the segmentation process (step 2 of the procedure). The correct perception of plant leaves 

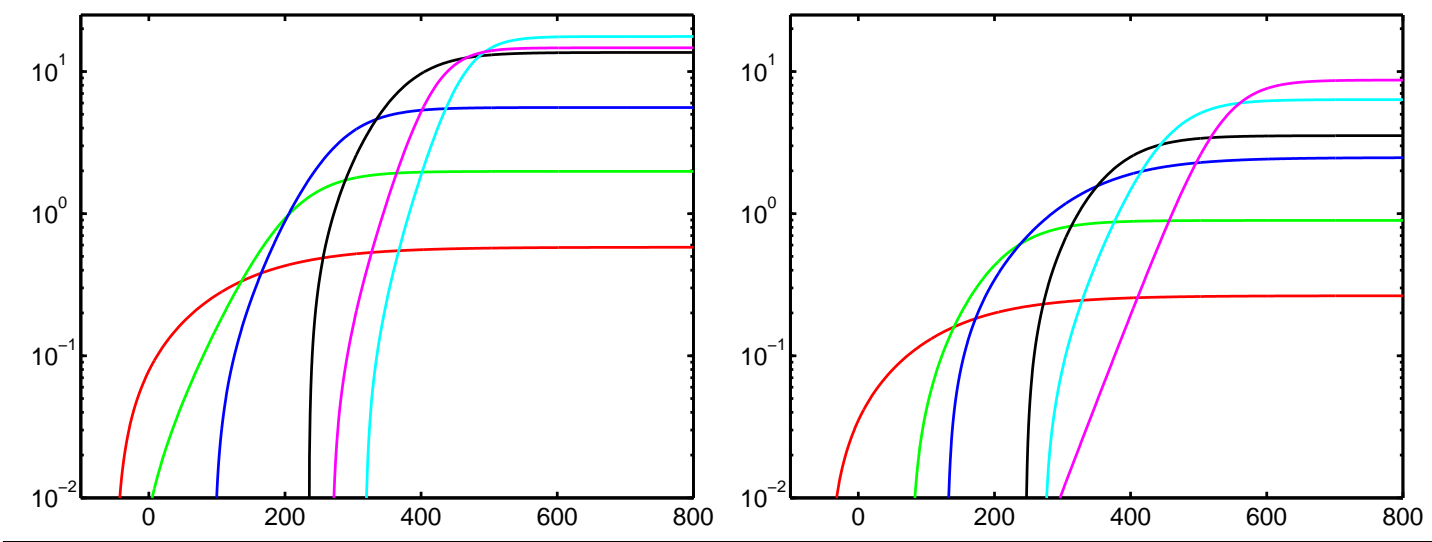

\begin{tabular}{|c|c|c|c|c|c|c|c|c|}
\hline & $\begin{array}{c}\text { Leaf } \\
\text { no. }\end{array}$ & $\begin{array}{c}\text { Max. } \\
\text { leaf } \\
\text { size } \\
A_{\infty}\left[\mathrm{cm}^{2}\right]\end{array}$ & $\begin{array}{l}\text { Growth } \\
\text { rate } \\
g r \\
{[\% / h]}\end{array}$ & $\begin{array}{l}\text { time } \\
\text { offset } \\
\qquad \begin{array}{c}\tau \\
{[\mathrm{h}]}\end{array}\end{array}$ & $\begin{array}{c}\text { size } \\
\text { offset } \\
A_{\text {offset }} \\
{\left[\mathrm{cm}^{2}\right]}\end{array}$ & $\begin{array}{c}\text { time } t_{c} \\
\text { at size } \\
c=0.1 \mathrm{~cm}^{2} \\
{[\mathrm{~h}]}\end{array}$ & $\begin{array}{c}\text { phyllo- } \\
\text { chron } \\
\text { [h] }\end{array}$ & $\begin{array}{c}\text { growth } \\
\text { duration } \\
\tau-t_{c} \\
{[\mathrm{~h}]}\end{array}$ \\
\hline \multirow{6}{*}{ 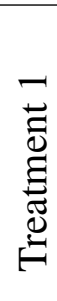 } & $1 \& 2$ & 0.8 & 1.0 & 66 & 0.17 & 12 & 0 & 54 \\
\hline & 3 & 2.0 & 2.3 & 205 & 0.01 & 80 & 68 & 125 \\
\hline & 4 & 5.7 & 2.3 & 267 & 0.10 & 126 & 46 & 141 \\
\hline & 5 & 14.5 & 2.2 & 356 & 0.90 & 240 & 114 & 116 \\
\hline & 6 & 14.8 & 3.5 & 417 & 0.08 & 292 & 52 & 125 \\
\hline & 7 & 17.7 & 3.6 & 458 & 0.12 & 335 & 43 & 123 \\
\hline \multirow{6}{*}{ 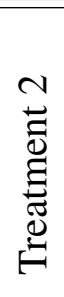 } & $1 \& 2$ & 0.3 & 1.1 & 62 & 0.08 & 72 & 0 & -10 \\
\hline & 3 & 1.0 & 2.1 & 195 & 0.08 & 123 & 51 & 72 \\
\hline & 4 & 2.8 & 1.3 & 294 & 0.32 & 155 & 32 & 139 \\
\hline & 5 & 3.9 & 2.1 & 352 & 0.37 & 258 & 103 & 94 \\
\hline & 6 & 6.4 & 2.5 & 446 & 0.08 & 304 & 46 & 142 \\
\hline & 7 & 8.7 & 2.9 & 533 & 0.00 & 377 & 72 & 156 \\
\hline
\end{tabular}

Figure 10: Fitted growth curves and parameters of autocatalytic model with constant offset $A_{\text {offset }}$ for all leaves. Left plot: Treatment 1. Right plot: Treatment 2. The vertical and horizontal axes represent leaf size $\left(\mathrm{cm}^{2}\right)$ and time (hours).

represents the most critical component of the procedure. In our framework, the superparamagnetic clustering of data has been chosen for the over-segmentation of leaves due to the following two reasons. First, this method accelerated on the GPU has a very high time performance and processes about 10 frames per second for image sizes of $640 \times 512$ pixels. 
Second, segments can be better merged by this algorithm using the convex hull approximation as compared to segments produced by conventional segmentation techniques such as the graph-based or mean shift technique from Felzenszwalb and Huttenlocher (2004); Comaniciu et al. (2002). This is because both of the latter techniques are dense, i.e, segments are forced to grow until all segments are larger than a minimum segment size. As a consequence, segments often grow into the small cavities that exist in the space between other segments, distorting the actual shape of segment, or can get more easily merged with other segments, as can be seen in the comparative Figure 11, where corrected segments for plant number 79339 using the graph-based segmentation (both middle columns) and superparamagnetic clustering of data (right column) within our framework are shown for selected frames.

In the graph-based approach the number of output segments is controlled by the threshold $k$ which should be lower than the recommended value $(k=500)$ to achieve the over-segmentation mode. We determined experimentally that $k=150$ guarantees oversegmentation for the majority of input frames (see the middle left column), while larger $k$ values can produce dramatic merges (see the middle right column). Overall, we obtained better results with the superparamagnetic clustering as compared to the graph-based technique.

We further analyzed how much the estimated number of leaves deviate from the ground truth provided, and compare the performance of the superparamagnetic clustering method with the one of the graph-based method Felzenszwalb and Huttenlocher (2004) when used inside our framework.

Figure 12 shows the comparison of the estimated number of leaves for three different 

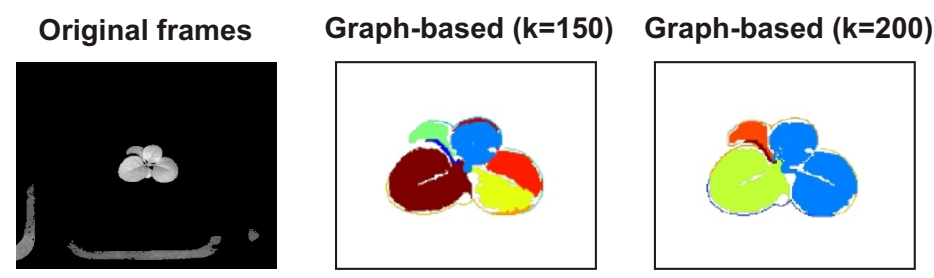

Superparamagnetic
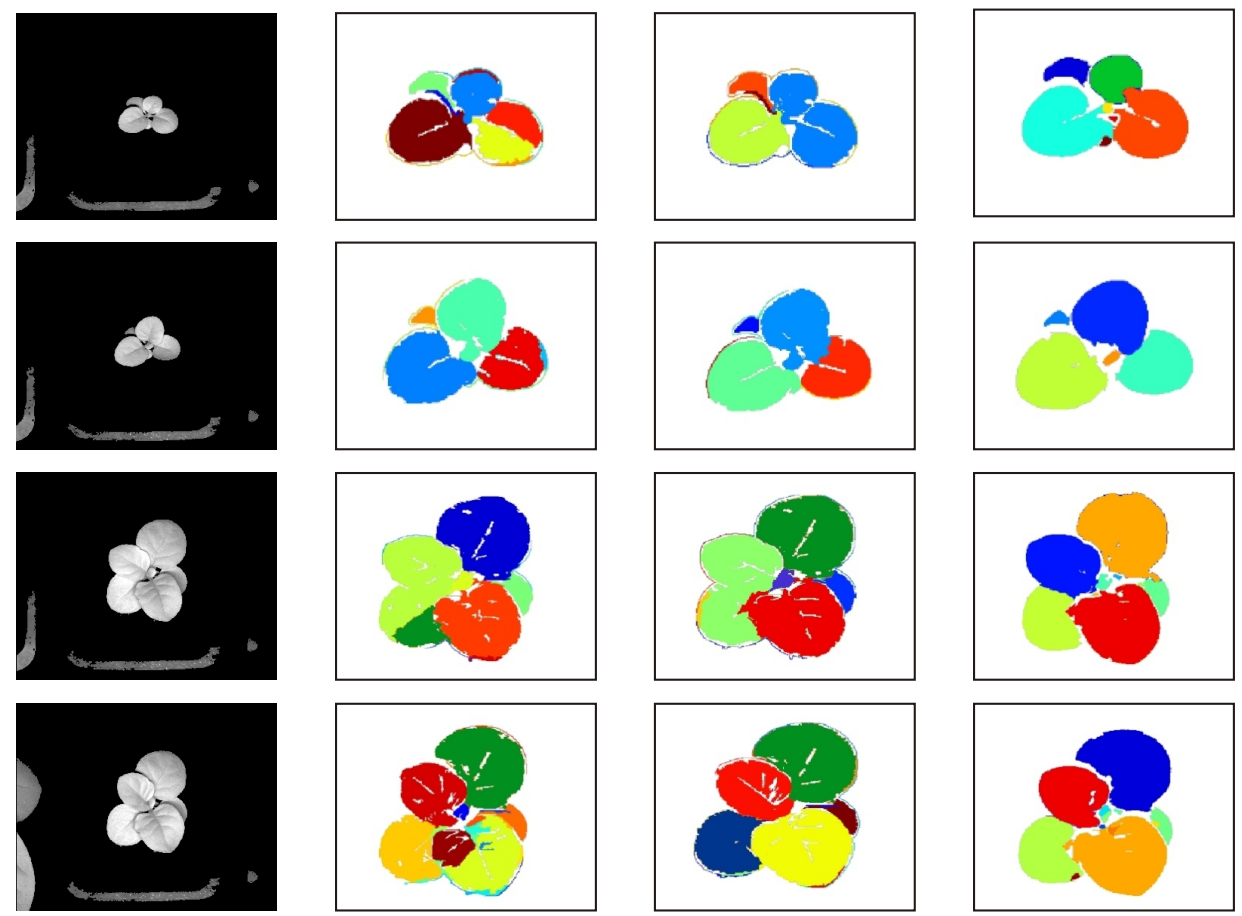

Figure 11: Leaf segmentation results obtained using the graph-based approach and the superparamagnetic clustering of data. Left column shows input near infrared (NIR) images for plant number 79339. Middle left and right columns show final segments for the graph-based method with threshold values $k=150$ and $k=200$, respectively. Segments from the superparamagnetic clustering are shown in the right column. Note that segments are here scaled up for a better visibility.

tobacco plants in the case of using the superparamagnetic clustering of data and the graphbased technique with the ground-truth data. The ground-truth data is obtained through human visual inspection, counting the number of leaves, including partially occluded ones. Both ground truth and the automatically computed number of leaves using our framework are shown for both segmentation approaches as a function of days. We can see that the number of leaves estimated with the superparamagnetic clustering agrees better with the ground truth than the graph-based method. However, both methods cannot handle the plant number 79336 after 25 days (see the high deviation between the estimated and actual 

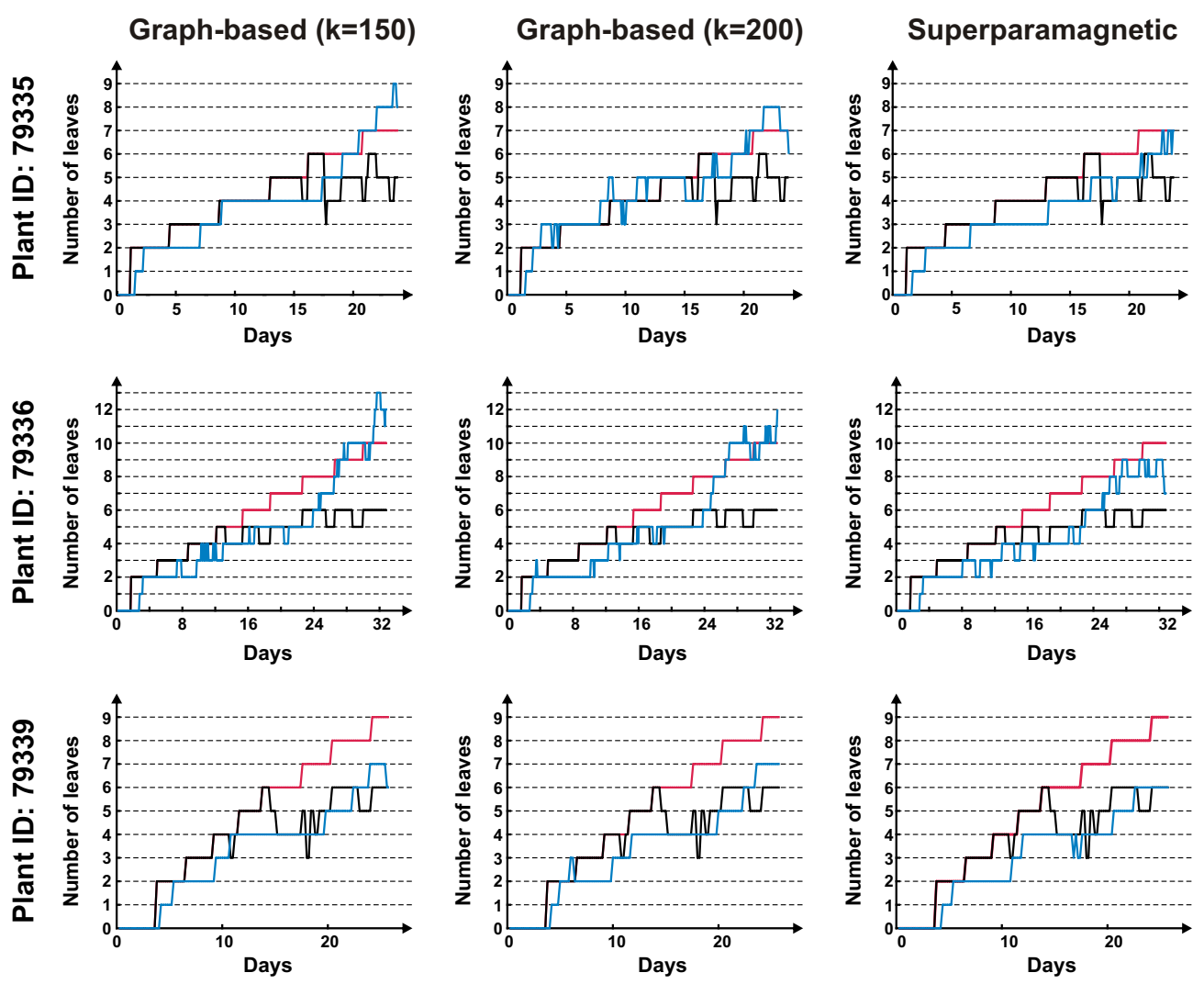

- Observable number of leaves

- Actual existing number of leaves

Estimated number of leaves

Figure 12: Comparison of the estimated number of leaves obtained for plant numbers 79335 (top row), 79336 (middle row), and 79339 (last row) using the graph-based approach and the superparamagnetic clustering of data for over-segmentation. The manually measured observable and actual existing number of leaves are used here as ground-truth data.

\begin{tabular}{cccc}
\hline Plant number & Graph-based $(k=150)$ & Graph-based $(k=200)$ & Superparamagnetic \\
\hline 79335 & 1.2230 & 1.3519 & 1.0021 \\
79336 & 2.0962 & 2.2180 & 1.4913 \\
79339 & 0.9883 & 0.9022 & 1.0268 \\
\hline
\end{tabular}

Table 1: The root-mean-square (RMS) error between the estimated and actual observed number of leaves for three different plants for the graph-based approach and the superparamagnetic clustering of data when used in our framework. 
observed number of leaves in Figure 12 (middle row). A quantitative evaluation of both methods with respect to the observable number of leaves based on the root-mean-square error is presented in Table 1 .

\section{Discussion}

The found average growth models are well in accordance with established literature.

Average per leaf growth rates of $2.5 \%$ (Treatment 1) or $2.0 \%$ (Treatment 2) are in the same range as the growth rates found in Walter and Schurr (1999). There, in Figure 1D, total leaf growth rates RGR between 12 and $18 \% /$ d, i.e. 0.5 and $0.75 \% / \mathrm{h}$, are reported together with the observation, that the biggest leaf contributes approx. $35 \%$ of the overall size and about 30 to $40 \%$ of the growth (Fig. 4B). As non-growing leaves are also taken into account for total leaf growth, growth rates for growing leaves need to be significantly higher than the averaging total, well in accordance with our findings.

Systematic increase of final leaf size $A_{\infty}$ of the first few leaves, as found for both treatments, are also reported in (Tsai et al., 1997, Figure 1). Absolute sizes are obviously treatment dependent, see Walter and Schurr (1999).

Phyllochron values reported in (Tsai et al., 1997, Figure 5, page 911) show a similar behavior as our findings. Leaf 4 emerges earlier than expected and leaf 5 somewhat later. The absolute duration between leaf emergence of the first 6 leaves lies however higher than under our treatments, i.e. between $72 \mathrm{~h}$ and $144 \mathrm{~h}$ with an average of approx. $110 \mathrm{~h}$ for a treatment with $300 \mu \mathrm{E} \mathrm{m}^{-2} \mathrm{~s}^{-1}$ photons and daily watering. Our treatments feature much higher light intensities and different watering strategies. Phyllochrons found here lie between $32 \mathrm{~h}$ and $114 \mathrm{~h}$ with averages of approx. $61 \mathrm{~h}$ or $65 \mathrm{~h}$, respectively. According 
to Munns (2002) leaf emergence rate is reduced under drought stress, thus clearly reacts to environmental conditions and thus differences found may be related to treatment differences.

The framework has been successfully applied inside a robot perception-action loop during experiments that were performed in the context of the EU project GARNICS. In these experiments, the robot had to make decisions about plant treatment based on sensory input, which was being processed with our multi-level pipeline, and water the plants accordingly. In the final experiments of the project the robot succeeded in taking care of the plants over a period of about three weeks, where the treatment found by the system resulted in a generally higher growth rate than in any of the training data.

\section{Conclusion}

We presented a novel multi-level procedure for finding and tracking of leaves of growing tobacco plants which allowed us to measure automatically important plant parameters, i.e., number of leaves and leaf size, as a function of time. The main challenge originates from the complex appearance of plants, making it difficult to segment plant organs. We used leaf-shape models to improve leaf segmentation and could successfully segment and track tobacco-plant leaves to up to an age of about 25 days. Beyond this growth stage, leaf segmentation turned out to be increasingly hard. As leaves grew older, we often observed under-segmentation errors. Fig. 13 shows examples where such under-segmentation effects have been observed. These problems can only be resolved by further improving the segmentation procedure.

The convex-hull approximation works well for tobacco plants but might have to be 
augmented using more sophisticated leaf models when dealing with other types of plants. The border detection as well as the depth reasoning could be improved in the future using e.g. a structured-light imaging system (Geng (2011)). The accuracy of the plant models estimated in Section 4.1 can further be improved by simply increasing the number of observed plants. Ellipses are used to estimate the size of the leaves from the segment boundaries in the last step of the algorithm. For tobacco plants, the ellipse model is an appropriate choice. For other plants, another leaf-shape model could be used instead of the ellipse. Assumptions about the leaf shape are also being made during the merging step (see Section 3.5). It is assumed that leaves have a convex shape. In some approximation, this holds for many types of plants, but it is not generally true. For non-convex leaf-shapes, the merging algorithm would have to be modified, and a specific leaf model could be fitted to the boundary of the object instead of finding its convex hull. Furthermore, we are currently analyzing plant vein structures which can then be used to correct segments and fit more accurate ellipses. Initial steps given in Johansson (2010) show promising results along this line.
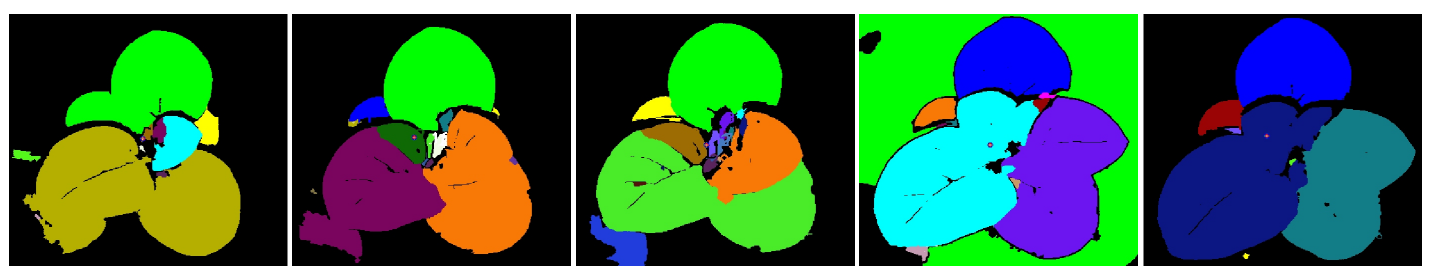

Figure 13: Under-segmentation errors observed once leaves are getting bigger. Merged segments have the same color. 


\section{Acknowledgements}

We thank Torge Herber from Forschungszentrum Jülich for the image acquisition.

The research leading to these results has received funding from the European Community's Seventh Framework Programme FP7/2007-2013 - Challenge 2 - Cognitive Systems, Interaction, Robotics - under grant agreement No 247947 - GARNICS. Babette Dellen acknowledges support from the Spanish Ministry for Science and Innovation through a Ramon y Cajal program.

\section{References}

A. Abramov, Pauwels K., J. Papon, F. Wörgötter, and B. Dellen. Real-time segmentation of stereo videos on a portable system with a mobile gpu. IEEE Transactions on Circuits and Systems for Video Technology, 9(22):1292-1305, 2012.

Guillem Aleny Ã, Babette Dellen, Sergi Foix, and Carme Torras. Robotized plant probing: Leaf segmentation utilizing time-of-flight data. IEEE Robot. Automat. Mag., 20(3):50$59,2013$.

G. Alenyà, B. Dellen, and C. Torras. 3d modelling of leaves from color and tof data for robotized plant measuring. Proc. IEEE Intl. Conf. on Robotics and Automation, 2011 a.

G. Alenyà, F. Moreno-Noguer, A. Ramisa, and C. Torras. Active perception of deformable objects using 3d cameras. In Workshop de Robotica Experimental, pages 434-440, Seville, $2011 b$.

B. Biskup, H. Scharr, U. Schurr, and U. Rascher. A stereo imaging system for measuring 
structural parameters of plant canopies. Plant, Cell and Environment, 30:1299-1308, 2007.

Andrew Blake and Andrew Zisserman. Visual Reconstruction. MIT Press, Cambridge, MA, USA, 1987. ISBN 0-262-02271-0.

G. Bradski. The OpenCV Library. Dr. Dobb's Journal of Software Tools, 2000.

Chung-Fang Chien, Yu-Che Cheng, and Ta-Te Lin. Robust ellipse detection based on hierarchical image pyramid and hough transform. Journal of the Optical Society of America, 28(4):581-589, 2011.

Dorin Comaniciu, Peter Meer, and Senior Member. Mean shift: A robust approach toward feature space analysis. IEEE Transactions on Pattern Analysis and Machine Intelligence, 24:603-619, 2002.

CORESTA CORESTA. A scale for coding growth stages in tobacco घ crops, Feb. 2009. URL http://wWw.coresta.org/Guides/ Guide-No07-Growth-Stages_Feb09.pdf

J. De Vylder, W. Philips, and D. Van Der Straeten. Multiple leaf tracking using computer vision methods with shape constraints. In Proc. of the International Conference on Sensing Technologies for Biomaterial, Food, and Agriculture (SPIE: SeTBio2013), Yokohoma, Japan, 2013.

Jonas De Vylder, Daniel Ochoa Donoso, Wilfried Philips, Laury Chaerle, and Dominique Van Der Straeten. Leaf segmentation and tracking using probabilistic parametric active 
contours. In A Gagalowizc and Wilfried Philips, editors, Lecture Notes in Computer Science, volume 6930, pages 75-85. Springer, 2011.

Pedro F. Felzenszwalb and Daniel P. Huttenlocher. Efficient graph-based image segmentation. International Journal of Computer Vision, 59(2):167-181, 2004.

Martin A. Fischler and Robert C. Bolles. Random sample consensus: A paradigm for model fitting with applications to image analysis and automated cartography. Commun. ACM, 24(6):381-395, June 1981. ISSN 0001-0782. doi: 10.1145/358669.358692.

Jason Geng. Structured-light 3d surface imaging: a tutorial. Adv. Opt. Photon., 3(2): 128-160, Jun 2011.

S. L. Horowitz and T. Pavlidis. Picture Segmentation by a directed split-and-merge procedure. Proceedings of the 2nd International Joint Conference on Pattern Recognition, Copenhagen, Denmark, pages 424-433, 1974.

Jian Jin and Lie Tang. Corn plant sensing using real-time stereo vision. Journal of Field Robotics, 26(6-7):591-608, 2009.

Peter Johansson. Plant condition measurement from spectral reflectance data. Master's thesis, Linköping University, Computer Vision, 2010.

Watcharin Kaewapichai and Pakorn Kaewtrakulpong. Robust ellipse detection by fitting randomly selected edge patches. World Academy of Science, Engineering and Technology, pages 30-33, 2008. 
H. Knutsson and CF. Westin. Normalized and differential convolution: Methods for interpolation and filtering of incomplete and uncertain data. In CVPR'93, pages 515-523, New York City, USA, 1993.

B.I. Loch, J.A. Belward, and J.S.Hanan. Application of surface fitting techniques for the representation of leaf surfaces. MODSIM 2005 International Congress on Modelling and Simulation, pages 1272-1278, 2005.

T. B. Moeslund, M. Aagaard, and D. Lerche. 3d pose estimation of cactus leaves using an active shape model. In Application of Computer Vision, 2005. WACV/MOTIONS '05 Volume 1. Seventh IEEE Workshops on, volume 1, pages 468-473, 2005.

R. Munns. Comparative physiology of salt and water stress. Plant, Cell and Environment, 25(2):239-250, 2002.

G. Polder, G.W.A.M. van der Heijden, H. Jalink, and J.F.H. Snel. Correcting and matching time sequence images of plant leaves using penalized likelihood warping and robust point matching. Computers and Electronics in Agriculture, 55(1):1 - 15, 2007. ISSN 0168-1699.

L. Quan, P. Tan, G. Zeng, L. Yuan, J. Wang, and S.B. Kang. Image-based plant modelling. ACM Siggraph, pages 599-604, 2006.

F. J. Richards. A flexible growth function for empirical use. Journal of Experimental Botany, 10(2):290-301, June 1959. doi: 10.1093/jxb/10.2.290.

L.O.L.A. Silva, M.L. Koga, C.E. Cugnasca, and A.H.R. Costa. Comparative assess- 
ment of feature selection and classification techniques for visual inspection of pot plant seedlings. Computers and Electronics in Agriculture, 97(0):47 - 55, 2013.

Ge Song and Hong Wang. A fast and robust ellipse detection algorithm based on pseudorandom sample consensus. In Proceedings of the 12th International Conference on Computer Analysis of Images and Patterns, pages 669-676, 2007.

Y. Song, R. Wilson, R. Edmondson, and N. Parsons. Surface modelling of plants from stereo images. 6th IEEE Intl. Conf. on 3D Digital Imaging and Modelling, 2007.

Ch.-H. Teng, Y.-T. Kuo, , and Y.-S. Chen. Leaf segmentation, classification, and threedimensional recovery from a few images with close viewpoints. Optical Engineering, 50(3), 2011. doi: 10.1117/1.3549927.

C. H. Tsai, A. Miller, M. Spalding, and S. Rodermel. Source strength regulates an early phase transition of tobacco shoot morphogenesis. Plant Physiol, 115(3):907-914, 1997.

Marcus Wallenberg, Michael Felsberg, Per-Erik Forssén, and Babette Dellen. Leaf segmentation using the kinect. In Proceedings of SSBA 2011 Symposium on Image Analysis, 2011.

A. Walter and U. Schurr. The modular character of growth in nicotiana tabacum plants under steady-state nutrition. Journal of Experimental Botany, 50(336):1169-1177, 1999. doi: $10.1093 / \mathrm{jxb} / 50.336 .1169$.

Jianlun Wang, Jianlei He, Yu Han, Changqi Ouyang, and Daoliang Li. An adaptive thresholding algorithm of field leaf image. Computers and Electronics in Agriculture, 96(0): 23 - 39, 2013. ISSN 0168-1699. 
545 F. Wörgötter, A. Abramov, E. E. Aksoy, and B. Dellen. Method and device for estimating 546 development parameters of plants. Patent office WO, Patent number 2013083146, 2013. 